DOI:

10.1038/nri2147

URLs

ATM

http://www.ncbi.nlm.nih.gov/ sites/entrez?Db=gene\&Cmd= ShowDetailView\&TermToSearc $\mathrm{h}=11920$ \&ordinalpos $=5$ \&itool $=$ EntrezSystem2.PEntrez.Gene. Gene_ResultsPanel.Gene_. RVDocSum

AID

http://www.ncbi.nlm.nih.gov/ sites/entrez?Db=gene\&Cmd= ShowDetailView\&TermToSearc $\mathrm{h}=11628$ \&ordinalpos $=2$ \&itool $=$ EntrezSystem2.PEntrez.Gene. Gene_ResultsPanel.Gene_ RVDocSum
...ATM has a

crucial role in

maintaining

genomic

stability by

activating

cell-cycle

checkpoints

in response to

DNA damage.

\title{
LYMPHOCYTE DEVELOPMENT
}

\section{Damage limitation}

During their development, all lymphocytes undergo a potentially dangerous process of purposefully introducing double-strand breaks (DSBs) in their genome with the ultimate aim of recombining their antigen-receptor genes for diverse antigen recognition. Only under the watchful eye of numerous factors do these breaks recombine appropriately, thereby protecting us from oncogenic chromosomal translocations. In this study, Callén et al. show that the absence of one such factor, the kinase ataxia-telangiectasia mutated (ATM), allows chromosome breaks and aberrant chromosome translocations that arise in developing lymphocytes to persist and propagate in mature lymphocytes, indicating that ATM has a crucial role in maintaining genomic stability by activating cell-cycle checkpoints in response to DNA damage.

ATM was recently shown to facilitate the repair of DSBs that are introduced into the DNA by the endonucleases RAG1 (recombination-activating gene 1) and RAG2 during lymphocyte antigen-receptor recombination. Subsequently, DSBs are also induced in the immunoglobulin heavy chain (IgH) locus by AID (activation-induced cytidine deaminase) in mature B cells undergoing antibody class switching a process that similarly involves ATM and other DNA-damage surveillance proteins. Accordingly, $\mathrm{Atm}^{-/-} \mathrm{B}$ cells have been shown to have high levels of IgH-associated breaks and chromosome translocations, compared with wild-type B cells. But when do these breaks actually occur?
To address this, the authors generated mice deficient in both ATM and AID, and induced class switching in B cells from these mice with lipopolysaccharide and interleukin-4. Cytogenetic analysis showed that only a subset of $\mathrm{IgH}$ associated chromosome translocations in $\mathrm{Atm}^{-/-} \mathrm{B}$ cells originated from AID-dependent DNA damage during class switching, indicating that DNA damage was probably generated in pre-replicative precursor $\mathrm{B}$ cells during recombination. Indeed, IgH-associated DSBs were detected in resting peripheral $\mathrm{Atm}^{-1-}$ $B$ cells and even in pre- $B$ cells from the bone marrow, at similar levels to those found in mature splenic $B$ cells. The authors also noted that DSBs in all these B-cell subsets from $\mathrm{Atm}^{-/-}$(and $\mathrm{Atm}^{-1-} \mathrm{Aid}^{-1-}$ ) mice commonly resulted in chromosomes that lack telomeres.

The authors then extended their studies to T cells and showed that telomere-deleted chromosome ends and T-cell receptor $\alpha$-chain (TCR $\alpha$ )associated translocations also accumulated in mature $\mathrm{Atm}^{-/-} \mathrm{T}$ cells, and that these aberrations similarly arose in immature $\mathrm{Atm}^{-1-} \mathrm{T}$-cell precursors. Consistent with this, antigen-receptor-associated chromosomal lesions in both $\mathrm{Atm}^{-/-} \mathrm{B}$ and $\mathrm{T}$ cells were shown to depend on RAG-mediated DNA cleavage during $\mathrm{V}(\mathrm{D}) \mathrm{J}$ recombination.

The finding that DSBs and terminally deleted chromosomes arose in immature lymphocytes and remained unresolved throughout lymphocyte development in the absence of ATM suggested that
DSBs are stable in vivo and that there was no selection against cells harbouring chromosome breaks. This was confirmed by showing that DSBs were retained in stimulated $\mathrm{Atm}^{-/-}$lymphocytes that had undergone several divisions, as assessed by dilution of the fluorescent dye CFSE (5,6-carboxyfluorescein diacetate succinimidyl ester).

Last, by taking advantage of a specific inhibitor of ATM kinase activity, the authors addressed the effect of restoring ATM activity after the accumulation of antigen-receptorassociated breaks. Stimulation of wild-type B cells in the presence of the ATM inhibitor led to the accumulation of chromosome aberrations, as seen in $\mathrm{Atm}^{-/-} \mathrm{B}$ cells, indicating that the kinase activity of ATM is essential for maintaining IgH stability. On removal of the inhibitor, the number of $\mathrm{B}$ cells with chromosome aberrations decreased, concomitant with the increase in ATM activity. This indicates that, in addition to its role in DNA repair, ATM activates a cell-cycle checkpoint in response to antigenreceptor-associated DSBs and that without these complementary functions DNA damage is unresolved and persists.

Importantly, DSBs that arise in the absence of ATM may participate in translocation reactions with chromosomes damaged in subsequent generations and contribute to the transformation of mature lymphocytes. This may explain the occurrence of IgH-associated translocations in mantle cell lymphoma, which is often associated with mutations in ATM.

Lucy Bird

ORIGINAL RESEARCH PAPER Callén, E. et al.

ATM prevents the persistence and propagation of chromosome breaks in lymphocytes. Cell 130, 63-75 (2007) 